\title{
Universidad y proyectos de vinculación con la sociedad, un análisis técnico legal y su efecto en los procesos de aprendizajes
}

Fecha de recepción : 22 de octubre de 2019 • Fecha de aceptación: 21 de febrero de 2019 • Fecha de publicación: 10 de mayo de 2020

Vicente Rodrigo Tamayo Arellano'

Universidad de Valladolid, España

viche.tamayo@hotmail.com

https://orcid.org/0000-0002-8057-2812

Carlos Wilfrido Taco Taco ${ }^{2}$ Universidad Estatal de Bolívar

cwtaco@ueb.edu.ec

https://orcid.org/0000-0002-4471-4975

Danny Fabián Hallo Montesdeoca ${ }^{3}$ Pontificia Universidad Católica del Ecuador - Sede Ambato

dhallo@pucesa.edu.ec

https://orcid.org/0000-0002-0315-8669

Graciela Maribel Fajardo Aguilar ${ }^{4}$ Universidad Técnica de Machala gfajardo@utmachala.edu.ec

https://orcid.org/0000-0003-1152-6414

\section{Resumen}

En el presente estudio se plasman principalmente los procesos de vinculación que mantienen las instituciones de educación superior con su entorno o la sociedad donde mantengan un enfoque ya sea económico, social, por sector o educativo y está regido por las políticas, leyes o normativas a nivel universitario. Para llevar a cabo el presente trabajo de investigación se realizó una comparación 
de la situación interna de Ecuador, con otros países de Latinoamérica y Europa en las instituciones de educación superior, por ello se busca saber si los fundamentos técnicos legales aportan positiva o negativamente al proceso de aprendizaje mediante una revisión de tipo documental descriptiva. Esta se hizo por medio de búsqueda electrónica en libros, tesis, revistas, ponencias y publicaciones de congresos afines al tema, desde sus inicios e información relevante y actualizada. Una de las concluyentes es que una institución de educación superior pretende cumplir en su totalidad con la normativa, ya sea interna o externa, en relación a la vinculación con la sociedad, buscando así el aprendizaje bidireccional, es decir, tanto de docentes y alumnos, como también el transmitir conocimiento a una comunidad o sector beneficiario.

\title{
Palabras clave: educación superior, normativas, vinculación, sociedad, universidad.
}

\begin{abstract}
This study reflects the processes of linking higher education institutions with their environment or society where they maintain an economic, social, sector or educational focus is maintained and is governed by the approach policies, laws or regulations to university level. To carry out this research, a comparison was made of the internal situation of Ecuador, with other countries in Latin America and Europe in higher education institutions, therefore, it is sought to know if the legal technical foundations contribute positively of or negatively to the learning process, through a descriptive documentary review. This was done through electronic search in books, theses, magazines, papers and publications of congresses in order to the topic of research since its inception and relevant and updated information. One of the conclusive is that a higher education institution intends to fully comply with the regulations, whether internal or external, in relation to the relationship with society, thus seeking bidirectional learning, that is, both teachers and students, as well as transmitting knowledge to a beneficiary community or sector.
\end{abstract}

Keywords: higher education, regulations, linking, society, university. 


\section{Introducción}

La relación o vinculación que mantienen las universidades con el entorno, ya sea social, económico, regional y local, desde la perspectiva de las políticas, leyes o normativas de educación superior a nivel global buscan mejorar la calidad en el ámbito académico y laboral, por lo que, se ha reformado los estatutos y políticas de la vinculación con la sociedad manteniendo así indicadores verificables del cumplimiento de la normativa (Jara, 2017). Por esto se considera a las Universidades Europeas como punto fuerte de comparación en función a la Ley Orgánica de Calidad de la Educación (LOCE), la cual, busca promover la mejora de la calidad en función a cinco ejes (Rajoy, 2013). Desde la perspectiva latinoamericana, un punto central y de relevancia es la situación en la educación en Argentina, por lo que la Secretaria de Políticas Universitarias reforzó la misión de las universidades con la sociedad y encontró la necesidad de fortalecer la construcción del conocimiento (Di Meglio y Harispe, 2015).

Por otro lado, las Instituciones pretenden diseñar diversas estrategias de vinculación con la sociedad, docentes y estudiantes, entonces, se espera que exista un efecto en los procesos de aprendizajes por lo que el Ecuador se maneja bajo el régimen de la Ley Orgánica de Educación superior (LOES).

Estupiñan, Villamar, Campi, y Cadena (2018) menciona que las universidades mantienen un fuerte impacto social como institución, donde los aspectos cognitivos, investigativos y académicos se complementan con problemas sociales buscando así soluciones para los mismos. De esta manera, se considera a la vinculación, docencia e investigación como un modelo Triple Hélice, por lo que existe una dependencia de una a la otra en función al cumplimiento de la gestión universitaria.

La importancia de la vinculación con la sociedad radica en la responsabilidad universitaria, por lo que, la proporción de experiencia en el proceso del conocimiento se aprecia desde diversas perspectivas, buscando favorecer al proceso de aprendizaje, ya que así se inserta nuevos conocimientos en el ámbito social (Rivas, Ángel, y López, 2017).

Este proceso interactivo, en función al entorno y proceso de aprendizaje, permite el desarrollo de proceso de formación a partir de las experiencias vividas; es decir, los estudiantes seleccionan, ordenan e integran diferentes hechos, y así establecen un vínculo con un sector (Acosta, Becerra, y Jaramillo, 2017).

La vinculación social parte de la pertenencia, la Organización de las Naciones Unidas para la Educación, la Ciencia y la Cultura (UNESCO) describe que la pertinencia de la educación superior debe considerarse en relación al lugar y a la sociedad, en otras palabras, en relación a la educación, docencia e investigación. Abarca aspectos cómo la relación de trabajo y en la relación con el estado y el financiamiento con la interacción y las diferentes formas de enseñanza (UNESCO, 1998). En la Conferencia Regional sobre políticas y estrategias para la Transformación de la Educación Superior en América Latina y El Caribe, lo cual conceptualiza a la pertenencia en la función que desempeña y el lugar donde se encuentra la educación superior en relación a las necesidades y demandas de sectores sociales. 
En una institución de educación superior, la pertinencia tiene como finalidad plantear objetivos dentro de un proyecto que beneficie a la sociedad (Ketele, 2008).

\section{Análisis técnico de la vinculación con la sociedad}

Las instituciones universitarias mantienen autonomía, por lo que poseen una estructura independiente interna, de forma externa se rife a normativas, leyes o reglamentos que normalizan las acciones universitarias.

En lo mencionado por la Asamblea Nacional en el año 2010, se manifiesta en La Ley Orgánica de Educación Superior en el artículo 8 en los literales d y h de los Fines en la Educación Superior, formar profesionales responsables y capaces de aportar al desarrollo de las instituciones educativas del Ecuador estimulando la participación social. Se pretende aportar al desarrollo nacional y local de forma continua por medio del trabajo o extensión comunitaria.

Mientras que en el artículo 87 se describe los requisitos previos a la obtención del título, los y las estudiantes deberán acreditar servicios a la comunidad mediante prácticas o pasantías pre profesionales, debidamente monitoreadas, en los campos de su especialidad, de conformidad con los lineamientos generales definidos por el Consejo de Educación Superior (CES) (CEAACES, 2018).

En el artículo 82, régimen académico, detalla a la vinculación con la sociedad y se enfoca en programas de educación superior continua, desarrollo y cooperación que permitan el avance de la innovación, este articulo dispone la potestad a las instituciones educativas establecer y gestionar la vinculación con la sociedad a fin de generar programas específicos que sean de interés. Por ejemplo, en Ecuador la Universidad Central de la ciudad de Quito ha estipulado un Plan Estratégico de Desarrollo Institucional, el cual involucra los procesos de prácticas pre profesionales, educación continua y diversos proyectos comunitarios.

\section{Revisión de los reglamentos de vinculación en universidades ecuatorianas}

Con un ejemplo claro y especifico se toma en consideración al plan Estratégico de la Universidad Central del Ecuador, la cual, en conjunto con la dirección de vinculación con la sociedad mantiene su normativa legal para el cumplimiento del programa planteado en el artículo 1, en el que se describe a la vinculación con la sociedad a las actividades académicas, culturales, científicas y de cooperación para la comunidad, donde las carreras deben gestionar en conjunto con la investigación y los docentes, con el fin de interactuar, instituciones ya sean públicas y privadas $\mathrm{u}$ organizaciones que promuevan el bien social por medio de proyectos dirigidos a promover el servicio y solucionar problemas estableciendo o redefiniendo conocimientos o prácticas que logran obtener los futuros profesionales en su formación académica (Universidad Central del Ecuador, 2018).

\subsection{Universidades de Categoría A y B}


En el aspecto legal y técnico de la vinculación se describen los estatutos planteados en las diferentes universidades, tanto de categoría A, como de categoría B, por lo que, en las dos categorías hay concordancia en el sustento para desarrollar políticas institucionales, las cuales están direccionadas para el fortalecer y direccionar actividades enlazadas a la vinculación con la sociedad, como se evidencio en la revisión literaria en una aproximación del $67 \%$ de las normas relacionadas a la vinculación de universidades son cumplidas, debido al análisis comparativo entre la universidad San francisco de Quito perteneciente a la categoría A y la Universidad Técnica de Ambato correspondiente a la categoría B, ver Tabla 1 (Murillo, Albarrasin, Bonilla, Caicedo, y Ramirez, 2017).

Tabla 1.

Comparación de universidades de categoría $A$ y $B$

\begin{tabular}{|c|c|c|c|}
\hline & Estrategia & Categoría A & Categoría B \\
\hline & Enunciado & San Francisco & $\begin{array}{l}\text { Universidad } \\
\text { Técnica de } \\
\text { Ambato }\end{array}$ \\
\hline 1 & $\begin{array}{l}\text { Mantienen apoyo para planteamiento de políticas dirigidas al } \\
\text { fomento del cumplimiento de actividades de vinculación con la } \\
\text { sociedad. }\end{array}$ & $X$ & $\mathrm{X}$ \\
\hline 2 & $\begin{array}{l}\text { Profundiza la unión de la institución de educación superior con } \\
\text { la sociedad, por medio del uso de la tecnología y la aplicación } \\
\text { de conocimientos, aportando así la resolución de conflictos de la } \\
\text { sociedad y su efecto en el desarrollo de la productividad en conjunto } \\
\text { de la participación de los miembros universitarios. }\end{array}$ & $X$ & \\
\hline 3 & $\begin{array}{l}\text { Impulsar acciones de vinculación con el adecuado proceso } \\
\text { para el aporte a la sociedad, con la inducción de programas } \\
\text { interdisciplinarios y multidisciplinarios, que aporten para el desarrollo } \\
\text { productivo y social. }\end{array}$ & $X$ & $x$ \\
\hline 4 & $\begin{array}{l}\text { Inducir la participación de instituciones de educación superior a } \\
\text { cubrir las necesidades identificadas en una sociedad. }\end{array}$ & $\mathrm{X}$ & $x$ \\
\hline \multicolumn{4}{|l|}{5} \\
\hline & $\begin{array}{l}\text { Fomentar el cambio de actitud en la institución para direccionar } \\
\text { la responsabilidad universitaria en relación a la sociedad en el } \\
\text { cumplimiento de actividades. }\end{array}$ & $x$ & $x$ \\
\hline \multicolumn{4}{|l|}{6} \\
\hline & $\begin{array}{l}\text { Obtener el apoyo de una comunidad beneficiarias, con el objetivo de } \\
\text { trabajar en conjunto para la solución de problemas. }\end{array}$ & $x$ & $x$ \\
\hline \multicolumn{4}{|l|}{7} \\
\hline & $\begin{array}{l}\text { Cumplimiento en la solución de problemas sociales, formando } \\
\text { actitudes de armonía en la institución de educación superior por } \\
\text { medio del capital humano y la optimización de recursos. }\end{array}$ & $x$ & $x$ \\
\hline \multicolumn{4}{|l|}{8} \\
\hline & $\begin{array}{l}\text { Generalizar los conocimientos y el acceso del mismo a las } \\
\text { comunidades para mejorar por medio de los conocimientos la } \\
\text { calidad de vida, y así, construir conocimientos. }\end{array}$ & $\mathrm{X}$ & $X$ \\
\hline 9 & $\begin{array}{l}\text { Relacionar a la institución de educación superior con comunidades } \\
\text { identificadas que no cuenten un acceso libre a información. }\end{array}$ & $x$ & $x$ \\
\hline 10 & $\begin{array}{l}\text { Asignar responsables para mantener una gestión operativa } \\
\text { previamente estructurada con la sociedad y su vinculación. }\end{array}$ & & $x$ \\
\hline
\end{tabular}


11 Implementar estrategias para todas las áreas que generen conocimiento como son los objetivos UC y PNBV, con la finalidad de $\quad X$ guiar las actividades de la vinculación en una sola dirección.

12 Plantear estrategias con el fin de identificar el efecto positivo de la $\quad X$ vida universitaria y su consecuencia en la sociedad.

\begin{tabular}{llll}
\hline 13 & $\begin{array}{l}\text { Identificar las necesidades de una comunidad o sociedad nacional, } \\
\text { regional o incluso local, y sugerir proyectos para cubrir necesidades } \\
\text { identificadas. }\end{array}$ & $\mathrm{X}$ & $\mathrm{X}$ \\
\hline 14 & Cumplir con el requisito de pasantías o prácticas Pre profesionales. & $\mathrm{X}$ & $\mathrm{X}$ \\
\hline 15 & $\begin{array}{l}\text { Fomentar la formación académica por medio de actividades de } \\
\text { educación continua y eventos tanto académicos como científicos. }\end{array}$ & $\mathrm{X}$ & \\
\hline 16 & Brindar servicios de asesoría o consultoría
\end{tabular}

Fuente: Información obtenida de la vinculación con la colectividad universitaria, casos y estrategias (Murillo, Albarrasin, Bonilla, Caicedo, y Ramirez, 2017).

En la Tabla 2 se evidencia un análisis de vinculación con la sociedad en comparación entre la: Universidad Europea y Universidad Ecuatoriana de categoría A.

Tabla 2.

Comparación entre universidades (Universidad Europea y Universidad Ecuatoriana de categoría A)

\begin{tabular}{llll}
\hline & Estrategia & Universidades & \\
\cline { 2 - 4 } & Enunciado & Universidad europea & $\begin{array}{c}\text { Universidad } \\
\text { ecuatoriana }\end{array}$ \\
\cline { 3 - 4 } & & $\begin{array}{l}\text { University } \\
\text { College London }\end{array}$ & Francisco \\
\hline 1 & Mantener relaciones estratégicas & $\mathrm{X}$ & $\mathrm{X}$ \\
\hline 2 & Invertir en cambio & $\mathrm{X}$ & $\mathrm{X}$ \\
\hline 3 & Cuenta con un centro dirigido para investigación & $\mathrm{X}$ \\
\hline 4 & Fomentar el emprendimiento y la innovación de la comunidad & $\mathrm{X}$ & $\mathrm{X}$ \\
\hline 5 & universitaria. & Se intercambia conocimientos entra la institución educativa, & $\mathrm{X}$ \\
\hline 6 & gobiernos y organizaciones. & $\mathrm{X}$ & \\
\hline 7 & Existen programas universitarios que aporten a la innovación. & $\mathrm{X}$ & $\mathrm{X}$ \\
\hline 8 & Mantiene una responsabilidad social cooperativa. & $\mathrm{X}$ & \\
\hline
\end{tabular}

Fuente: Información obtenida de la vinculación con la colectividad universitaria, casos y estrategias (Murillo, Albarrasin, Bonilla, Caicedo, y Ramirez, 2017).

\subsection{Estado de aprendizaje}


Según lo descrito por Zapataros (2015), el proceso de aprendizaje necesita diversos entornos y enfoques, por lo que señala que una institución educativa no depende únicamente de la infraestructura o tecnología, si no que el aprendizaje depende de la capacidad del individuo para crear, adquirir, aplicar y distribuir los conocimientos. El entorno para el aprendizaje (ver Tabla 3) es donde el estudiante buscar resolver las actividades, se empodera de sucesos y de su accionar, desde la perspectiva del docente es el lugar donde se transmite y procesa el aprendizaje mediante la aplicación de estrategias, donde se estimula la investigación y donde se deben optimizar los recursos (Beltrán, Iñigo, y Mata, 2014).

Tabla 3.

Entornos del aprendizaje

\begin{tabular}{ll}
\hline Entorno & Descripción \\
\hline Aprendizaje físico & Espacio donde existe la interacción entre e docente y estudiante. \\
\hline Aprendizaje formal & $\begin{array}{l}\text { Espacios para aprendizajes específicos, adaptados para asignaturas } \\
\text { específicas. }\end{array}$ \\
\hline Aprendizaje virtual & $\begin{array}{l}\text { Espacios que cuenten con equipos necesarios para transmitir infor- } \\
\text { mación. }\end{array}$ \\
\hline Aprendizaje abierto & $\begin{array}{l}\text { Espacio donde se desarrolla varias actividades y donde existe flexibil- } \\
\text { idad en el proceso de aprendizaje. }\end{array}$ \\
\hline Aprendizaje a distancia & $\begin{array}{l}\text { Participación individual a cargo del docente o tutor, se maneja una } \\
\text { comunicación unidireccional, pero cuenta con el uso de tecnología. }\end{array}$ \\
\hline Aprendizaje presencial & Espacios de participación activa. \\
\hline
\end{tabular}

Fuente: Información obtenida de efectos del modelo de vinculación con la sociedad de la facultad de comunicación social de la universidad central del ecuador, desde la perspectiva del entorno de aprendizaje, 2017.

\section{Metodología}

Se realizo una revisión de tipo documental descriptiva, por medio de la revisión y búsqueda electrónica en libros, tesis, revistas, ponencias y publicaciones de congresos afines al tema de investigación, desde sus inicios y aspectos relevantes, así como también se tomó información actualizada entre los años 2014 y 2018, por medio de fuentes de información como Google académico, revista electrónicas como Scielo, Latindex, Ciencia y Trabajo, Interacoes, Revista Orbita Pedagógica y base de datos como Redalyc, Dialnet y ResearchGate.

Se obtuvieron 15 publicaciones que cumplieron con los criterios de búsqueda, incluyendo a los autores pioneros del tema de investigación, hasta las publicaciones más actuales, por lo que las referencias fueron redactadas de acuerdo al estilo American Psychological Association (APA), derivado de que es una forma estructurada para organizar la información, desde su obtención hasta el registro planteado en el documento generado por el trabajo realizado. 


\section{Resultados}

\section{Los resultados obtenidos de los principales documentos como fuentes de información son evidenciados en la Tabla 4.}

Tabla 4.

Resultados de revisión bibliográfica

\begin{tabular}{|c|c|c|c|c|}
\hline Autor (año y revista) & País & $\begin{array}{l}\text { Tipo de } \\
\text { estudio }\end{array}$ & $\begin{array}{l}\text { Origen } \\
\text { Resultado }\end{array}$ & Medida del resultado \\
\hline $\begin{array}{l}\text { Acosta, L., Becerra, } \\
\text { F., y Jaramillo, } \\
\text { D. Formación } \\
\text { Universitaria, } 2017 .\end{array}$ & Ecuador & $\begin{array}{l}\text { Revisión } \\
\text { bibliográfica }\end{array}$ & $\begin{array}{l}\text { Investigación de revisión } \\
\text { bibliográfica es exploratoria, } \\
\text { ya que su búsqueda fue } \\
\text { basada en los sistemas } \\
\text { de información y la } \\
\text { aplicación a nivel nacional e } \\
\text { internacional. }\end{array}$ & $\begin{array}{l}\text { En los procesos de educación } \\
\text { superior, la gestión se maneja un } \\
\text { área vinculada a los sistemas de } \\
\text { información, estrategias utilizadas } \\
\text { para la vinculación universitaria, } \\
\text { apoyo, evaluaciones, planificación y } \\
\text { planificación. }\end{array}$ \\
\hline $\begin{array}{l}\text { CEAACES } \\
2018\end{array}$ & Ecuador & Normativa & $\begin{array}{l}\text { Ley Orgánica De Educación } \\
\text { Superior, Loes (Secretaría) }\end{array}$ & $\begin{array}{l}\text { Fundamentación del cumplimiento } \\
\text { de la vinculación con la sociedad de } \\
\text { instituciones de educación superior. }\end{array}$ \\
\hline $\begin{array}{l}\text { Di Meglio, M. } \\
\text { F., y Harispe, A. } \\
\text { Interações (Campo } \\
\text { Grande), } 2015\end{array}$ & Argentina & $\begin{array}{l}\text { Estudio de } \\
\text { caso }\end{array}$ & $\begin{array}{l}\text { Se mantuvo un registro de } \\
\text { documentación de fuentes } \\
\text { primarias. }\end{array}$ & $\begin{array}{l}\text { Se obtuvo sobre resoluciones y la } \\
\text { fundamentación para investigación } \\
\text { y la vinculación que mantiene las } \\
\text { universidades en función a los } \\
\text { convenios realizado. }\end{array}$ \\
\hline $\begin{array}{l}\text { Estupiñan, J., } \\
\text { Villamar, M., Campi, } \\
\text { A., \& Cadena, L. } \\
\text { Revista Órbita } \\
\text { Pedagógica, } 2018 .\end{array}$ & Ecuador & $\begin{array}{l}\text { Revisión } \\
\text { bibliográfica }\end{array}$ & Sistematización de teorías & $\begin{array}{l}\text { Se identifica desde conceptos clases } \\
\text { hasta la responsabilidad social de } \\
\text { universidades y la calidad de la } \\
\text { misma. }\end{array}$ \\
\hline $\begin{array}{l}\text { Ketele, J. Revista } \\
\text { de Curriculum y } \\
\text { Formación Del } \\
\text { Profesorado, } 2008 .\end{array}$ & España & $\begin{array}{l}\text { Revisión } \\
\text { bibliográfica }\end{array}$ & Evolución del conocimiento & Proceso de enseñanza. \\
\hline $\begin{array}{l}\text { Murillo, F., } \\
\text { Albarrasin, N., } \\
\text { Bonilla, D., Caicedo, } \\
\text { F., y Ramirez, } \\
\text { W. II Congreso } \\
\text { Universidad } \\
\text { Ecuador, } 2017 .\end{array}$ & Ecuador & $\begin{array}{l}\text { Análisis de } \\
\text { casos }\end{array}$ & $\begin{array}{l}\text { Aspectos técnicos y legales } \\
\text { de la vinculación en la } \\
\text { educación superior }\end{array}$ & $\begin{array}{l}\text { Comparación entre el cumplimiento } \\
\text { técnico legal de universidades } \\
\text { nacionales e internacionales. }\end{array}$ \\
\hline $\begin{array}{l}\text { Jara, N. Universidad } \\
\text { Autonoma de los } \\
\text { Andes (2017) }\end{array}$ & Ecuador & Investigación & $\begin{array}{l}\text { Métodos teóricos y métodos } \\
\text { empíricos de enseñanza }\end{array}$ & Métodos de enseñanza y aprendizaje. \\
\hline $\begin{array}{l}\text { Rajoy, M. STECyL-I, } \\
2013 .\end{array}$ & España & Normativa & Ley Orgánica 8/2013 & $\begin{array}{l}\text { Aspectos de aprendizaje para la guía } \\
\text { de la calidad en la educación. }\end{array}$ \\
\hline $\begin{array}{l}\text { Rivas, A., Ángel, } \\
\text { M., y López, M. } \\
\text { Revista de Ciencias } \\
\text { de Seguridad y } \\
\text { Defensa, } 2017 .\end{array}$ & Ecuador & Investigativo & $\begin{array}{l}\text { Normativa interna para } \\
\text { el cumplimiento de la } \\
\text { vinculación con la sociedad } \\
\text { de la UCE. }\end{array}$ & $\begin{array}{l}\text { Conocer la normativa interna del } \\
\text { cumplimiento de la vinculación con } \\
\text { la sociedad de una universidad } \\
\text { ecuatoriana. }\end{array}$ \\
\hline
\end{tabular}

Fuente: elaboración propia 
En Ecuador existe un progreso considerable a la vinculación con la sociedad universitaria, por lo que las instituciones de educación superior están cambiando su rumbo a contribuir a las soluciones de problemas identificando las necesidades, en el cumplimiento de la normativa legal en el Ecuador y acatando lo estipulado en la Constitución. En la Tabla 5 se muestra una comparación entre la Ley Orgánica de Educación Superior y el Reglamento de Régimen Académico, basándose en los artículos más representativos para la revisión.

Tabla 5.

Cumplimiento de las universidades en el aspecto técnico legal

\begin{tabular}{|c|c|}
\hline Régimen & Normativa \\
\hline $\begin{array}{l}\text { CONSTITUCION DE } \\
\text { LA REPUBLICA DEL }\end{array}$ & $\begin{array}{l}\text { CONSTITUCION DE LA REPUBLICA DEL ECUADOR } \\
2008\end{array}$ \\
\hline ECUADOR 2008 & $\begin{array}{l}\text { Art. 425. "La Constitución; los tratados y convenios } \\
\text { internacionales; las leyes orgánicas; las leyes } \\
\text { ordinarias; las normas regionales y las ordenanzas } \\
\text { distritales; los decretos y reglamentos; las ordenanzas; } \\
\text { los acuerdos y las resoluciones; y los demás actos y } \\
\text { decisiones de los poderes públicos". }\end{array}$ \\
\hline
\end{tabular}

Ley Orgánica de

Educación Superior

Ley Orgánica de Educación Superior

Art. 88. "Servicios a la comunidad. - Para cumplir con la obligatoriedad de los servicios a la comunidad".

Art. 107.- "Principio de pertinencia. - El principio de pertinencia consiste en que la educación superior responda a las expectativas y necesidades de la sociedad".

Art. 125.- "Programas y cursos de vinculación con la sociedad. - Las instituciones del Sistema de Educación Superior realizarán programas y cursos de vinculación con la sociedad guiados por el personal académico.

Para ser estudiante de los mismos no hará falta cumplir los requisitos del estudiante regular".

Art. 127. "Otros programas de estudio. - Las universidades y escuelas politécnicas podrán realizar en el marco de la vinculación con la colectividad, cursos de educación continua y expedir los correspondientes certificados".

\section{Reglamento de CES (2016)}

Régimen Académico

Artículo 78.- "Fortalezas o dominios académicos de las instituciones de educación superior. - Un dominio académico consiste en las fortalezas científicas, tecnológicas, humanísticas y artísticas demostradas por una IES, con base en su trayectoria académica e investigativa, 78 personal académico altamente calificado".

\section{Cumplimiento}

Se generaliza el acceso a la información y conocimientos, transmitiéndolos a comunidades adquiriendo así una mejor calidad de vida, generando la construcción adecuada de conocimientos.

Acceso a pasantías o practicas preprofesionales.

Relación que tiene las instituciones educativas tiene las instituciones con sectores que no cuentan con acceso al conocimiento por medio de capacitaciones e información. Fomento de la innovación y el emprendimiento de la institución.

Cuenta con un centro dirigido para investigación. Intercambio de información entre gobiernos, instituciones e instituciones educativas.

Fuente: Elaboración propia a partir de La vinculación con la colectividad universitaria, casos y estrategias, 2017.

Por lo que al señalar los indicadores en función a la normativa se identifica que en la formación y actualización se realiza una formación continua por medio de eventos académicos y científicos.

El ambiente o entorno de aprendizaje (ver Tabla 6), considerado abiertos, se adaptan de forma flexible identificando su función de facilitar el aprendizaje, debido a que es una estructura definida del proceso de aprendizaje, por lo que, la misma estructura será la base del aprendizaje y no de 
forma rígida, condicionando el significado de los contenidos y de las interpretaciones de los mismo (Rivas et al., 2017).

Tabla 6.

Entornos den aprendizaje abierto

\begin{tabular}{lll}
\hline Momento & Lugar de ejecución & Resultados \\
\hline Académico & Espacios (Aula) & Aprendizaje y el empoderamiento. \\
\hline Proceso & $\begin{array}{l}\text { Espacio (Aula abierta y vinculación con la } \\
\text { sociedad) }\end{array}$ & Aporte a la sociedad \\
\hline Producto & Espacio (Aula y Comunidad) & $\begin{array}{l}\text { Solución al problema comunitario } \\
\text { identificado. }\end{array}$ \\
\hline
\end{tabular}

Fuente: Información obtenida de efectos del modelo de vinculación con la sociedad de la facultad de comunicación social de la universidad central del ecuador, desde la perspectiva del entorno de aprendizaje, 2017.

\section{Conclusiones}

Al realizar una revisión bibliográfica se identificó información relevante sobre la comparación entre instituciones de educación superior en Latinoamérica, como punto central en Ecuador se contrapone a la universidad más reconocida de categoría $A$ y de categoría $B$, se tomó como referencia una institución de educación superior de Europa y se buscó referencias del régimen legal como punto focal la normativa ecuatoriana.

En la relación de universidades y en respuesta a que, si los fundamentos técnicos legales de la educación superior aportan al proceso de aprendizaje, en función a la revisión de literatura se análisis casos donde se evidencia que al gestionar proyectos de vinculación las universidades se fundamentan en la normativa legal tanto externa como interna aportando al proceso de aprendizaje.

En Latinoamérica o en Europa existe un objetivo global el cual es resolver problemas sociales y de desarrollo productivo, con la participación activa y organizada de los miembros de la comunidad universitaria para beneficio de una comunidad o grupo identificado en base a una necesidad.

La vinculación con la sociedad universitaria busca tener acceso a los conocimientos, así como también, transferir estos a la comunidad, vinculando en muchos casos a las universidades a sectores donde no cuenten con acceso a capacitaciones e información, por lo que uno de los puntos más evaluados en el cumplimiento de la vinculación con la sociedad es el promover el conocimiento como se identificó en la comparación de universidades nacionales con europeas.

Debido a que la formación debe ser continua por medio de actividades científicas y académicas, la vinculación en el proceso de aprendizaje parte desde el entorno de la formación en el aspecto académico, ya sea abierto o cerrado (aula de clase) se fomenta el aprendizaje y el empoderamiento cuando se maneja en los dos ambientes. 
Al revisar la documentación encontrada se verifica que el cumpliendo del proceso entre la vinculación con el aula abierta y la sociedad genera un aporte significativo al aprendizaje de la sociedad y finalmente el producto por medio del aula y la comunidad es la solución o aporte otorgado por la institución a la comunidad. 


\section{Referencias bibliográficas}

Acosta, L., Becerra, F., y Jaramillo, D. (2017). Sistema de Información Estratégica para la Gestión Universitaria en la Universidad de Otavalo (Ecuador). Formación Universitaria, 10(2), 103-112. https://doi.org/10.4067/ S0718-50062017000200011

Beltrán, J., Iñigo, E., \& Mata, A. (2014). La responsabilidad social universitaria, el reto de su construcción permanente. Revista Iberoamericana de Educación Superior, 5(14), 3-18. https://doi.org/10.1016/s20072872(14)70297-5

CEAACES. (2018). Ley Organica De Educacion Superior, Loes (Secretaría). Quito: SENPLADES.

Di Meglio, M. F., y Harispe, A. (2015). Estrategias institucionales de "vinculación universitaria" orientadas al desarrollo local. Reflexiones a partir de un estudio de caso. Interações (Campo Grande), 16(1), $203-217$. https://doi.org/10.1590/1518-70122015117

Estupiñan, J., Villamar, M., Campi, A., y Cadena, L. (2018). Reflexiones acerca de la pertinencia e impacto de la educación superior en Ecuador desde su perspectiva actual. Revista Órbita Pedagógica, 3(3), 81-92.

Jara, N., \& Fabian, C. (2017). La destitución de los servidores públicos en las entidades educativas de nivel superior, frente al derecho constitucional de recurrir.

Ketele, J. de. (2008). Enfoque socio-histórico de las competencias en la enseñanza. Revista de Curriculum y Formación Del Profesorado, 13.

Murillo, F., Albarrasin, N., Bonilla, D., Caicedo, F., \& Ramirez, W. (2017). La vinculacion con la colectividad universitaria, casos y estrategias. II Congreso Universidad Ecuador, 584-593.

Rajoy, M. (2013). Ley Orgánica 8/2013, de 9 de diciembre, para la mejora de la calidad educativa. STECyL-I, 91.

Rivas, A., Ángel, M., y López, M. (2017). Sociedad de la facultad de comunicación social de la universidad central del Ecuador, desde la perspectiva del entorno de aprendizaje. Revista de Ciencias de Seguridad y Defensa, 2(1), 47-56.

Unesco. (1998). Informe mundial sobre la educación: los docentes y la enseñanza en un mundo en mutación. Unesco 1998.

Universidad Central del Ecuador, U. (2018). Plan Estratégico de Desarrollo Institucional de la Universidad Central del Ecuador 2018-2022. Universidad Central del Ecuador. Quito.

Zapataros, M. (2015). Teorías y modelos sobre el aprendizaje en entornos conectados y ubicuos. Eks, 16(1), 69-102. https://doi.org/10.14201/eks201516169102 
Copyright (c) 2020 Vicente Rodrigo Tamayo Arellano, Carlos Wilfrido Taco Taco, Danny Fabián Hallo Montesdeoca y Graciela Maribel Fajardo Aguilar



Este texto está protegido bajo una licencia internacional Creative Commons 4.0.

Usted es libre para Compartir - copiar y redistribuir el material en cualquier medio o formato - y Adaptar el documento - remezclar, transformar y crear a partir del material-para cualquier propósito, incluso para fines comerciales, siempre que cumpla las condiciones de Atribución. Usted debe dar crédito a la obra original de manera adecuada, proporcionar un enlace a la licencia, e indicar si se han realizado cambios. Puede hacerlo en cualquier forma razonable, pero no de forma tal que sugiera que tiene el apoyo del licenciante o lo recibe por el uso que hace de la obra.

$\underline{\text { Resumen de licencia - Texto completo de la licencia }}$ 